\title{
Comparison of i-gel, LMA-supreme, LMA-classic and LMA- proseal as conduits of endotracheal intubation in newborns and infants: A manikin study
}

\author{
Aysun Ankay-Yılbaş ${ }^{1}$, Betül Başaran², Filiz Üzümcügil ${ }^{1}$, Başak Akçâ1, Murat İzgi ${ }^{1}$, \\ Özgür Canbay ${ }^{1}$ \\ ${ }^{1}$ Department of Anesthesiology and Reanimation, Hacettepe University, Faculty of Medicine, Ankara; ${ }^{2}$ Clinics of Anesthesiology \\ and Reanimation, Konya Training and Research Hospital, Konya, Turkey.E-mail: aysunankay@hotmail.com \\ Received: 29th November 2018, Accepted: 04th January 2019
}

SUMMARY: Ankay-Yılbaş A, Başaran B, Üzümcügil F, Akça B, İzgi M, Canbay Ö. Comparison of i-gel, LMA-supreme, LMA-classic and LMA-proseal as conduits of endotracheal intubation in newborns and infants: A manikin study. Turk J Pediatr 2019; 61: 166-173.

Many types of supraglottic airway devices (SAD) including the traditional LMA (Laryngeal Mask Airway) are commonly used as conduits for intubation in pediatric patients with difficult airway. The aim of this study was to evaluate the feasibility of four types of commonly used neonatal and infant sized SADs as conduits of intubation. Fiberoptic-guided tracheal intubation with uncuffed, cuffed and armored uncuffed endotracheal tubes (ETT) sized between 2.5 and 4.5 through four commonly used types of size 1 and 1.5 SADs (i-gel, LMA-classic, LMA-supreme, LMA-proseal) were performed by two investigators on an infant manikin. The investigators scored two main outcomes with a 5-point scale: 1) passage of ETT during intubation through the SAD, and 2) passage of SAD over the ETT during SAD removal. The differences between the study groups were evaluated using the Bonferroniadjusted Mann-Whitney $U$ test and $p<0.0083$ was considered as statistically significant according to Bonferroni correction. i-gel sizes 1 and 1.5 both performed better as conduits for fiberoptic-guided intubation compared with LMA-proseal, LMA-classic and LMA-supreme with most of the uncuffed ETTs investigated $(\mathrm{p}<0.0083)$. We found i-gel sizes 1 and 1.5 easily feasible to use even with uncuffed ETTs with an inner diameter of $3.5 \mathrm{~mm}$ and $4.5 \mathrm{~mm}$, respectively. i-gel was the only SAD that was feasible for use as a conduit for armored ETTs. The passage of cuffed ETTs was problematic with all types of studied SADs. In conclusion; the choice of i-gel as a conduit for intubation could be safer than LMA-classic, LMA-supreme and LMA-proseal.

Key words: supraglottic airway device, difficult airway, newborn, infant.

Difficult airway management is much more challenging in newborns and infants due to having limited apnea time to significant hypoxemia and increased risk of iatrogenic injuries with repeated intubation attempts. ${ }^{1}$ The guideline developed by the Delphi Group with the support of the Association of Paediatric Anaesthetists of Great Britain and Ireland, and the Difficult Airway Society recommends supraglottic airway devices (SAD) as the most appropriate second-line devices in cases of unanticipated difficult airway in children aged 1 to 8 years. ${ }^{2}$ Besides providing a rescue technique for ventilation, SADs could be used as a conduit for easy and safe endotracheal intubation guided by a flexible fiberoptic bronchoscope (FOB). One fiberoptic intubation attempt via SAD is also recommended in the same guideline in children if the SAD is satisfactorily placed and the child is stable with appropriate muscle relaxation. ${ }^{2}$ The opportunity of ventilation throughout the intubation process to minimize the risk of oxygen desaturation and the relief of upper airway obstruction are the major benefits of this technique. ${ }^{3,4}$ The presence of a SAD could additionally provide 
a guide for the FOB and diminish blurring of the vision due to blood and secretions. ${ }^{5}$

There are specially designed SADs that act as conduits for intubation, however many types of SADs including the traditional LMA (Laryngeal Mask Airway) have been used with success for this purpose for years. ${ }^{3,6,7}$ The main restriction of the technique is the difficulty to pass the endotracheal tube through the SAD, especially in newborns and infants. Modifications such as an additional step with an exchange catheter or a guidewire may be required to overcome this problem. However, data regarding the experience of using SADs as conduits of intubation in newborns and infants are still limited. ${ }^{4,8}$ The aim of this study was to evaluate the feasibility of four types of commonly used neonatal and infant sized SADs as conduits of intubation.

\section{Material and Methods}

We designed an in vitro study to evaluate the convenience of different neonatal and infant sized SADs for the passage and removal of endotracheal tubes. The study was performed in Hacettepe University, Faculty of Medicine, Department of Anesthesiology and Reanimation, Ankara, Turkey. The study was conducted on an infant manikin (Life/form replicas, Nasco Fort Atkinson, Wisconsin, United States) designed for intubation training; therefore, ethical committee approval was not required.

\section{The procedure}

Two different investigators (AAY and FU) who are both experienced in pediatric anesthesia, pediatric difficult airway management and pediatric SAD inserted different types of SADs (sizes 1.0 and 1.5 for each type) into the manikin's pharynx following appropriate lubrication. After placement of the SAD with the standard midline insertion technique, each investigator inserted a pediatric $\mathrm{FOB}$ with an outer diameter of $2.8 \mathrm{~mm}$ and $3.0 \mathrm{~mm}$ (Karl Storz, GmbH\&Co., Tuttlingen, Germany) loaded with appropriate sized endotracheal tubes through the SAD and performed intubation on the manikin. The intubation attempts were performed through a catheter mount attached to the SAD to simulate a real situation. Lastly, the investigators removed the SADs and FOB from the pharynx leaving the ETT in the trachea of the manikin. When the proximal end of the ETT came to the same level as the end of the SAD, another similar size ETT was used to keep the ETT in place. After the ETT was placed into the trachea, the SAD was removed from the mouth and the ETT was grasped at level of the incisor teeth. Possible accidental dislodgement of the tracheal tube was tested using a FOB directly inside the ETT. The investigators were blinded to each other's results.

The investigators were asked to grade the feasibility of passage of ETT and SAD during both insertion of the ETT through the SAD and removal of the SAD with a 5-point scale: $0=$ very easy, $1=$ easy, $2=$ moderate, $3=$ hard, and $4=$ impossible.

The two main outcome parameters scored were 1) passage of endotracheal tube (ETT) during intubation through the SAD and 2) passage of the SAD over the ETT during SAD removal. Both investigators performed the same procedure 5 times every other day to score 5 different samples of each tested ETT and SAD.

\section{Investigated SADs and ETTS}

We investigated four different types of commonly used pediatric SADs, sized 1.0 and 1.5. The SADs studied included i-gel ${ }^{\circledR}$ (Intersurgical Ltd., Wokingham, Berkshire, UK), LMA-Supreme $^{\mathrm{TM}}$ (Teleflex, Westmeath, Ireland), classical LMA (reusable) (Teleflex, Westmeath, Ireland) and LMA ProSeal (Ningbo TianHou Import and Exp. Co., China).

The ETTs (Bicakcilar, Istanbul, Turkey) included were sized between 2.5 and 4.5 inner diameter (ID) and there were three types of ETTs (cuffed, uncuffed and armored uncuffed) used in the study. An FOB with an outer diameter of 2.8 $\mathrm{mm}$ was used for loading ETTs with an inner diameter (ID) of $3.0 \mathrm{~mm}$ and an FOB with an outer diameter of $3.0 \mathrm{~mm}$ was used for larger ETTs. If it was not feasible to intubate with an ETT with ID of $3.0 \mathrm{~mm}$ easily, then blind intubation with an ETT with an ID of $2.5 \mathrm{~mm}$ was tried for that SAD type.

\section{Statistical analysis}

Data analysis was performed by using IBM SPSS Statistics version 17.0 software (IBM Corporation, Armonk, NY, USA). Descriptive statistics for the feasibility of ETT insertion and the feasibility of SAD removal were shown as median $(25$ th -75 th) percentiles. Whether 
Table I. Evaluations for the Feasibility of Insertion of ETTs Through Size 1 SADs According to Both Observers.

\begin{tabular}{|c|c|c|c|c|c|c|c|c|c|c|}
\hline & \multirow{2}{*}{ i-gel } & \multirow{2}{*}{$\begin{array}{c}\text { LMA- } \\
\text { supreme }\end{array}$} & \multirow{2}{*}{$\begin{array}{l}\text { LMA- } \\
\text { classic }\end{array}$} & \multirow{2}{*}{$\begin{array}{l}\text { LMA- } \\
\text { proseal }\end{array}$} & \multicolumn{6}{|c|}{ Multiple comparisons $\dagger$} \\
\hline & & & & & $p^{1}$ & $p^{2}$ & $p^{3}$ & $p^{4}$ & $p^{5}$ & $p^{6}$ \\
\hline \multicolumn{11}{|l|}{$1^{\text {st }}$ observer } \\
\hline Normal, ID $2.5 \mathrm{~mm}$ & - & $3(2.5-3.5)$ & $2(0.5-2.5)$ & - & - & - & - & 0.056 & - & - \\
\hline Normal, ID $3.0 \mathrm{~mm}$ & $0(0-0)$ & $4(4-4)$ & $3(2-3)$ & $0(0-1.5)$ & 0.008 & 0.008 & 0.310 & 0.008 & 0.008 & 0.016 \\
\hline Armed, ID $3.0 \mathrm{~mm}$ & $0(0-0)$ & $4(4-4)$ & $4(4-4)$ & $3(3-3.5)$ & 0.008 & 0.008 & 0.008 & 0.999 & 0.032 & 0.032 \\
\hline Normal, ID $3.5 \mathrm{~mm}$ & $0(0-0.5)$ & - & $4(4-4)$ & $4(4-4)$ & - & 0.008 & 0.008 & - & - & 0.999 \\
\hline Armored, ID $3.5 \mathrm{~mm}$ & $1(0.5-1)$ & - & $4(4-4)$ & $4(4-4)$ & - & 0.008 & 0.008 & - & - & 0.999 \\
\hline Normal, ID $4.0 \mathrm{~mm}$ & $4(4-4)$ & - & - & - & - & - & - & - & - & - \\
\hline Armored, ID $4.0 \mathrm{~mm}$ & $4(4-4)$ & - & - & - & - & - & - & - & - & - \\
\hline \multicolumn{11}{|l|}{$2^{\text {nd }}$ observer } \\
\hline Normal, ID $2.5 \mathrm{~mm}$ & - & $3(2.5-3.5)$ & $2(0-2.5)$ & - & - & - & - & 0.056 & - & - \\
\hline Normal, ID $3.0 \mathrm{~mm}$ & $0(0-0)$ & $4(4-4)$ & $3(2-3)$ & $1(0-1)$ & 0.008 & 0.008 & 0.151 & 0.008 & 0.008 & 0.008 \\
\hline Armored, ID $3.0 \mathrm{~mm}$ & $0(0-0)$ & $4(4-4)$ & $4(4-4)$ & $4(4-4)$ & 0.008 & 0.008 & 0.008 & 0.999 & 0.999 & 0.999 \\
\hline Normal, ID $3.5 \mathrm{~mm}$ & $1(0-1)$ & - & $4(4-4)$ & $4(4-4)$ & - & 0.008 & 0.008 & - & - & 0.999 \\
\hline Armored, ID $3.5 \mathrm{~mm}$ & $1(1-1)$ & - & $4(4-4)$ & $4(4-4)$ & - & 0.008 & 0.008 & - & - & 0.999 \\
\hline Normal, ID $4.0 \mathrm{~mm}$ & $4(4-4)$ & - & - & - & - & - & - & - & - & - \\
\hline Armored, ID $4.0 \mathrm{~mm}$ & $4(4-4)$ & - & - & - & - & - & - & - & - & - \\
\hline
\end{tabular}

Data were shown as median $\left(25^{\text {th }}-75^{\text {th }}\right)$ percentiles, $\dagger$ Mann-Whitney $U$ test, according to the Bonferroni correction a p-value less than 0.0083 was considered as statistically significant, ${ }^{1}$ The comparisons between i-gel and LMA-supreme, ${ }^{2}$ The comparisons between i-gel and LMA-classic, ${ }^{3}$ The comparisons between i-gel and LMA-proseal, ${ }^{4}$ The comparisons between LMA-supreme and LMA-classic, ${ }^{5}$ The comparisons between LMA-supreme and LMA-proseal, ${ }^{6}$ The comparisons between LMAclassic and LMA-proseal.

ETT: endotracheal tube, ID: inner diameter, LMA: laryngeal mask airway, SAD: supraglottic airway devices

the differences in the process difficulty grades between the study groups (i.e. i-gel, LMA supreme, Classical LMA and LMA ProSeal) were statistically significant was evaluated using the Bonferroni-adjusted Mann-Whitney $\mathrm{U}$ test. According to the Bonferroni correction, p-values less than 0.0083 were considered as statistically significant. Kappa coefficients for the feasibility of ETT insertion and SAD removal were calculated to determine the interobserver agreement levels. A p-value less than 0.05 was considered statistically significant.

\section{Results}

Successful device placement was achieved on the first attempt with all SADs tested on the manikin. No accidental dislodgement of the ETT occurred when removal of the SAD was successful. The inter-observer agreement level was found significantly high for both insertion of the ETT through the SAD and removal of the SAD over the ETT $(p<0.001)$.

\section{Uncuffed ETTs}

Size 1 SADs: The data regarding the insertion of ETTs and removal of SADs for both investigators can be seen in Table I and Table II respectively.

The insertion of standard and armored ETTs with IDs of 3.0 and $3.5 \mathrm{~mm}$ were both managed easily by the two investigators through i-gel size 1 and the SAD was again easily removed over the ETT. i-gel was the only size 1 SAD through which it was possible to intubate with ETT with an ID of $3.5 \mathrm{~mm}$. It was not possible to insert any sizes of standard or armored type ETTs with FOB guidance through LMA-supreme size 1 by either of the two investigators.

i-gel was found significantly superior to other SADs in most of the measurements for both insertion of the ETT and removal of the SAD over the ETT (Table I, Table II, p<0.0082). i-gel was the only size 1 SAD feasible for the use of armored ETTs. In the case of standard ETTs with an ID of $3.0 \mathrm{~mm}$; although i-gel again 
Table II. Evaluations for the Feasibility of Removal of Size 1 SADs According to Both Observers.

\begin{tabular}{|c|c|c|c|c|c|c|c|c|c|c|}
\hline & \multirow{2}{*}{ i-gel } & \multirow{2}{*}{$\begin{array}{c}\text { LMA- } \\
\text { supreme }\end{array}$} & \multirow{2}{*}{$\begin{array}{l}\text { LMA- } \\
\text { classic }\end{array}$} & \multirow{2}{*}{$\begin{array}{l}\text { LMA- } \\
\text { proseal }\end{array}$} & \multicolumn{6}{|c|}{ Multiple comparisons $\dagger$} \\
\hline & & & & & $p^{1}$ & $p^{2}$ & $p^{3}$ & $p^{4}$ & $p^{5}$ & $p^{6}$ \\
\hline \multicolumn{11}{|l|}{$1^{\text {st }}$ observer } \\
\hline Normal, ID $2.5 \mathrm{~mm}$ & - & $3(2.25-3)$ & $2(0.5-3)$ & - & - & - & - & 0.286 & - & - \\
\hline Normal, ID $3.0 \mathrm{~mm}$ & $0(0-0)$ & - & $4(2.5-4)$ & $2(0.5-2)$ & - & 0.008 & 0.032 & - & - & 0.016 \\
\hline Armored, ID $3.0 \mathrm{~mm}$ & $0(0-0)$ & - & - & $4(4-4)$ & - & - & 0.016 & - & - & - \\
\hline Normal, ID $3.5 \mathrm{~mm}$ & $1(1-2)$ & - & - & - & - & - & - & - & - & - \\
\hline Armored, ID $3.5 \mathrm{~mm}$ & $1(1-2)$ & - & - & - & - & - & - & - & - & - \\
\hline Normal, ID $4.0 \mathrm{~mm}$ & - & - & - & - & - & - & - & - & - & - \\
\hline Armored, ID $4.0 \mathrm{~mm}$ & - & - & - & - & - & - & - & - & - & - \\
\hline
\end{tabular}

$2^{\text {nd }}$ observer

Normal, ID $2.5 \mathrm{~mm}$

$3(2.25-3)$

$2(0-3)$

-

$-$

Normal, ID $3.0 \mathrm{~mm}$

$0(0-0)$

$4(3-4)$

$1(0.5-2)$

$-0.008$

-
0.032
-
-
-
-

0.286

Armored, ID $3.0 \mathrm{~mm}$

$0(0-0)$

Normal, ID $3.5 \mathrm{~mm}$

$1(1-2)$

Armored, ID $3.5 \mathrm{~mm}$

$1(1-2)$

Normal, ID $4.0 \mathrm{~mm}$

Armored, ID $4.0 \mathrm{~mm}$

Data were shown as median $\left(25^{\text {th }}-75^{\text {th }}\right)$ percentiles, $\dagger$ Mann-Whitney $U$ test, according to the Bonferroni correction a p-value less than 0.0083 was considered as statistically significant, ${ }^{1}$ The comparisons between i-gel and LMA-supreme, 2 The comparisons between i-gel and LMA-classic, ${ }^{3}$ The comparisons between i-gel and LMA-proseal, ${ }^{4}$ The comparisons between LMA-supreme and LMA-classic, ${ }^{5}$ The comparisons between LMA-supreme and LMA-proseal, ${ }^{6}$ The comparisons between LMAclassic and LMA-proseal.

ETT: endotracheal tube, ID: inner diameter, LMA: laryngeal mask airway, SAD: supraglottic airway devices

seemed clinically more feasible compared with LMA-proseal, the difference was not statistically significant for ETT insertion or SAD removal.

Size 1.5 SADs: The data regarding the insertion of ETTs and removal of SADs for both investigators can be seen in Table III and Table IV respectively.

i-gel, LMA-proseal and LMA-supreme performed similarly as conduits for standard ETT with an ID of $3.0 \mathrm{~mm}$. i-gel and LMA-classic performed better than LMA-proseal and LMA-supreme as conduits for standard ETTs with IDs of 3.5 $\mathrm{mm}$. However, SAD removal was easier with i-gel compared with LMA-classic $(p<0.0082)$. i-gel size 1.5 was found feasible by both investigators to insert ETTs with IDs up to $4.5 \mathrm{~mm}$ through the SAD and to remove the SAD over the ETT.

For armored ETTs with an ID of $3.0 \mathrm{~mm}$, i-gel and LMA-classic were found significantly more feasible compared with LMA-proseal and LMAsupreme $(p<0.0082)$. i-gel was the only SAD feasible for armored ETTs with IDs between 3.5-4.5 mm.

\section{Cuffed ETTs}

It was possible to intubate with cuffed ETTs with an ID of $2.5 \mathrm{~mm}$ through a size 1 i-gel and all SADs of size 1.5. However, we could not remove any of the tested SADs after intubation with cuffed ETTs even with an ID of $2.5 \mathrm{~mm}$.

\section{Discussion}

The results of this manikin study showed that i-gel size 1 and 1.5 both performed better as conduits for fiberoptic-guided intubation compared with LMA-proseal, LMA-classic and LMA-supreme with most of the uncuffed ETTs investigated. i-gel was the only SAD that was found feasible as a conduit for armored ETTs. However, the passage of cuffed ETTs was problematic with all types of studied SADs.

Intubation-related complications especially as a result of hypoxemia are much more common in infants with difficult airway than older children. ${ }^{9}$ However, the literature regarding this population is usually limited due to ethical reasons and the difficulty of designing a 
Table III. Evaluations for the Feasibility of Insertion of ETTs Through Size 1.5 SADs According to Both Observers.

\begin{tabular}{|c|c|c|c|c|c|c|c|c|c|c|}
\hline & \multirow{2}{*}{ i-gel } & \multirow{2}{*}{$\begin{array}{c}\text { LMA- } \\
\text { supreme }\end{array}$} & \multirow{2}{*}{$\begin{array}{l}\text { LMA- } \\
\text { classic }\end{array}$} & \multirow{2}{*}{$\begin{array}{l}\text { LMA- } \\
\text { proseal }\end{array}$} & \multicolumn{6}{|c|}{ Multiple comparisons $\dagger$} \\
\hline & & & & & $p^{1}$ & $p^{2}$ & $p^{3}$ & $p^{4}$ & $p^{5}$ & $p^{6}$ \\
\hline \multicolumn{11}{|l|}{$1^{\text {st }}$ observer } \\
\hline Normal, ID $2.5 \mathrm{~mm}$ & - & $3(2.5-4)$ & - & - & - & - & - & - & - & - \\
\hline Normal, ID $3.0 \mathrm{~mm}$ & $0(0-0)$ & $4(4-4)$ & $0(0-0)$ & $0(0-0.5)$ & 0.008 & 0.999 & 0.690 & 0.008 & 0.008 & 0.690 \\
\hline Armored, ID $3.0 \mathrm{~mm}$ & $0(0-0)$ & $4(4-4)$ & $0(0-0)$ & $2(2-2)$ & 0.008 & 0.999 & 0.008 & 0.008 & 0.008 & 0.008 \\
\hline Normal, ID $3.5 \mathrm{~mm}$ & $0(0-0)$ & - & $0(0-0.5)$ & $3(3-3)$ & - & 0.690 & 0.008 & - & - & 0.008 \\
\hline Armored, ID $3.5 \mathrm{~mm}$ & $0(0-0)$ & - & $2(2-2)$ & $4(4-4)$ & - & 0.008 & 0.008 & - & - & 0.008 \\
\hline Normal, ID $4.0 \mathrm{~mm}$ & $0(0-0)$ & - & $4(4-4)$ & - & - & 0.008 & - & - & - & - \\
\hline Armored, ID $4.0 \mathrm{~mm}$ & $0(0-0)$ & - & $4(4-4)$ & - & - & 0.008 & - & - & - & - \\
\hline Normal, ID $4.5 \mathrm{~mm}$ & $1(0.5-1)$ & - & - & - & - & - & - & - & - & - \\
\hline Armored, ID $4.5 \mathrm{~mm}$ & $1(1-1.5)$ & - & - & - & - & - & - & - & - & - \\
\hline \multicolumn{11}{|l|}{$2^{\text {nd }}$ observer } \\
\hline Normal, ID $2.5 \mathrm{~mm}$ & - & $3(2.5-4)$ & - & - & - & - & - & - & - & - \\
\hline Normal, ID $3.0 \mathrm{~mm}$ & $0(0-0)$ & $4(4-4)$ & $0(0-0)$ & $0(0-0.5)$ & 0.008 & 0.999 & 0.690 & 0.008 & 0.008 & 0.690 \\
\hline Armored, ID $3.0 \mathrm{~mm}$ & $0(0-0)$ & $4(4-4)$ & $0(0-0)$ & $1(1-2)$ & 0.008 & 0.999 & 0.008 & 0.008 & 0.008 & 0.008 \\
\hline Normal, ID $3.5 \mathrm{~mm}$ & $0(0-0)$ & - & $0(0-0.5)$ & $3(3-3)$ & - & 0.690 & 0.008 & - & - & 0.008 \\
\hline Armored, ID $3.5 \mathrm{~mm}$ & $0(0-0)$ & - & $2(2-2)$ & $4(4-4)$ & - & 0.008 & 0.008 & - & - & 0.008 \\
\hline Normal, ID $4.0 \mathrm{~mm}$ & $0(0-0)$ & - & $4(4-4)$ & - & - & 0.008 & - & - & - & - \\
\hline Armored, ID $4.0 \mathrm{~mm}$ & $1(0.5-1)$ & - & $4(4-4)$ & - & - & 0.008 & - & - & - & - \\
\hline Normal, ID $4.5 \mathrm{~mm}$ & $1(1-1)$ & - & - & - & - & - & - & - & - & - \\
\hline Armored, ID $4.5 \mathrm{~mm}$ & $1(1-1.5)$ & - & - & - & - & - & - & - & - & - \\
\hline
\end{tabular}

Data were shown as median $\left(25^{\text {th }}-75^{\text {th }}\right)$ percentiles, $\dagger$ Mann-Whitney $U$ test, according to the Bonferroni correction a p-value less than 0.0083 was considered as statistically significant, ${ }^{1}$ The comparisons between i-gel and LMA-supreme, ${ }^{2}$ The comparisons between i-gel and LMA-classic, ${ }^{3}$ The comparisons between i-gel and LMA-proseal, ${ }^{4}$ The comparisons between LMA-supreme and LMA-classic, ${ }^{5}$ The comparisons between LMA-supreme and LMA-proseal, ${ }^{6}$ The comparisons between LMAclassic and LMA-proseal.

ETT: endotracheal tube, ID: inner diameter, LMA: laryngeal mask airway, SAD: supraglottic airway devices

controlled randomized study without giving rise to complications in this fastidious and vulnerable patient population. In a study of Burjek et al. ${ }^{9}$; fiberoptic intubation via SADs were associated with higher first-attempt success rates of intubation compared with video laryngoscopy in infants with difficult airway. Accordingly, SADs are widely used as conduits for tracheal intubation in the pediatric population because of their advantage of continuous oxygenation and relief of upper airway obstruction. ${ }^{4}$ Relief of upper airway obstruction and the provision of a better laryngeal view also facilitate the technique compared with unguided fiberoptic intubation, which requires more maneuvers and experience. ${ }^{10}$ Air-Q and Ambu Aura-i are among the SADs that are specially designed to assist tracheal intubation in children and infants. With its shorter and wider airway tube design air-Q seems to have advantages especially for the use of cuffed ETTs. ${ }^{4}$ In a study of Jagannathan et $\mathrm{al}^{11}$, i-gel also served as an effective conduit for intubation in children, similar to air- $Q$, in the hands of inexperienced trainees. However, more complications regarding device removal were seen with i-gel. ${ }^{11}$ i-gel also performed better as a conduit for intubation in our study compared with LMA-supreme, LMA-classic and LMA-proseal. We did not grade the laryngeal views; however, successful device placement at the first attempt and sufficient ventilation was achieved with all SADs tested. The fact that fiberoptic intubation was easily performed with i-gel compared with three other types of SADs in this study, could be thought consistent with former studies that showed better FOB grades of view with i-gel compared with LMA-classic and LMA-proseal. ${ }^{12,13}$ 
Table IV. Evaluations for the Feasibility of Removal of Size 1.5 SADs According to Both Observers.

\begin{tabular}{|c|c|c|c|c|c|c|c|c|c|c|}
\hline & \multirow[b]{2}{*}{ i-gel } & \multirow{2}{*}{$\begin{array}{c}\text { LMA- } \\
\text { supreme }\end{array}$} & \multirow{2}{*}{$\begin{array}{l}\text { LMA- } \\
\text { classic }\end{array}$} & \multirow{2}{*}{$\begin{array}{l}\text { LMA- } \\
\text { proseal }\end{array}$} & \multicolumn{6}{|c|}{ Multiple comparisons $\dagger$} \\
\hline & & & & & $p^{1}$ & $p^{2}$ & $p^{3}$ & $p^{4}$ & $p^{5}$ & $p^{6}$ \\
\hline \multicolumn{11}{|l|}{$1^{\text {st }}$ observer } \\
\hline Normal, ID $2.5 \mathrm{~mm}$ & - & $3(3-4)$ & - & - & - & - & - & - & - & - \\
\hline Normal, ID $3.0 \mathrm{~mm}$ & $0(0-0)$ & - & $0(0-0)$ & $0(0-1)$ & - & 0.999 & 0.690 & - & - & 0.690 \\
\hline Armored, ID $3.0 \mathrm{~mm}$ & $0(0-0)$ & - & $0(0-0)$ & $2(2-2)$ & - & 0.999 & 0.008 & - & - & 0.008 \\
\hline Normal, ID $3.5 \mathrm{~mm}$ & $0(0-0)$ & - & $1(1-2)$ & $3(3-3.5)$ & - & 0.008 & 0.008 & - & - & 0.032 \\
\hline Armored, ID $3.5 \mathrm{~mm}$ & $0(0-0)$ & - & $3(2-3)$ & - & - & 0.008 & - & - & - & - \\
\hline Normal, ID $4.0 \mathrm{~mm}$ & $0(0-0)$ & - & - & - & - & - & - & - & - & - \\
\hline Armored, ID $4.0 \mathrm{~mm}$ & $0(0-0)$ & - & - & - & - & - & - & - & - & - \\
\hline Normal, ID $4.5 \mathrm{~mm}$ & $1(1-2)$ & - & - & - & - & - & - & - & - & - \\
\hline Armored, ID $4.5 \mathrm{~mm}$ & $2(1.5-2.5)$ & - & - & - & - & - & - & - & - & - \\
\hline \multicolumn{11}{|l|}{$2^{\text {nd }}$ observer } \\
\hline Normal, ID $2.5 \mathrm{~mm}$ & - & $3(3-3)$ & - & - & - & - & - & - & - & - \\
\hline Normal, ID $3.0 \mathrm{~mm}$ & $0(0-0)$ & - & $0(0-0)$ & $0(0-1)$ & - & 0.999 & 0.690 & - & - & 0.690 \\
\hline Armored, ID $3.0 \mathrm{~mm}$ & $0(0-0)$ & - & $0(0-0)$ & $2(2-2)$ & - & 0.999 & 0.008 & - & - & 0.008 \\
\hline Normal, ID $3.5 \mathrm{~mm}$ & $0(0-0)$ & - & $1(1-2)$ & $3(3-3.5)$ & - & 0.008 & 0.008 & - & - & 0.032 \\
\hline Armored, ID $3.5 \mathrm{~mm}$ & $0(0-0)$ & - & $3(2.5-3)$ & - & - & 0.008 & - & - & - & - \\
\hline Normal, ID $4.0 \mathrm{~mm}$ & $0(0-0)$ & - & - & - & - & - & - & - & - & - \\
\hline Armored, ID $4.0 \mathrm{~mm}$ & $0(0-0)$ & - & - & - & - & - & - & - & - & - \\
\hline Normal, ID $4.5 \mathrm{~mm}$ & $1(1-2)$ & - & - & - & - & - & - & - & - & - \\
\hline Armored, ID $4.5 \mathrm{~mm}$ & $2(1.5-2.5)$ & - & - & - & - & - & - & - & - & - \\
\hline
\end{tabular}

Data were shown as median $\left(25^{\text {th }}-75^{\text {th }}\right)$ percentiles, $\dagger$ Mann-Whitney $U$ test, according to the Bonferroni correction a p-value less than 0.0083 was considered as statistically significant, ${ }^{1}$ The comparisons between i-gel and LMA-supreme, ${ }^{2}$ The comparisons between i-gel and LMA-classic, ${ }^{3}$ The comparisons between i-gel and LMA-proseal, ${ }^{4}$ The comparisons between LMA-supreme and LMA-classic, ${ }^{5}$ The comparisons between LMA-supreme and LMA-proseal, ${ }^{6}$ The comparisons between LMAclassic and LMA-proseal.

ETT: endotracheal tube, ID: inner diameter, LMA: laryngeal mask airway, SAD: supraglottic airway devices

The maximum size of ETTs recommended by the manufacturer were $3.0 \mathrm{~mm}$ and $4.0 \mathrm{~mm}$ for i-gel size 1 and 1.5 respectively. However, we found i-gel size 1 and 1.5 easily feasible for use, even with uncuffed ETTs with an ID of $3.5 \mathrm{~mm}$ and $4.5 \mathrm{~mm}$. The possibility to use larger size ETTs in appropriate conditions could be accepted as an advantage of i-gel to avoid additional maneuvers when exchanging the ETT. i-gel was also the only SAD that seemed feasible for use as a conduit for armored ETTs in this study. Armored ETTs could be useful in procedures on the head and neck such as cranioplasty, cleft lip and palate surgeries, and mandibular distractions in which bending or compression of the tube is possible. ${ }^{14}$ In the event of difficult intubation in these children, the feasibility of i-gel as a conduit for armored ETTs could provide an advantage.
Although there are not manufacturer recommendations for LMA-supreme, LMAclassic and LMA-proseal, they have been commonly used as conduits for intubation in children in cases when no special SADs were available. 5,7,15,16 Similarly, in our study, the feasibility of intubation with standard uncuffed $3.0 \mathrm{~mm}$ ETT through an LMA-proseal size 1 was statistically comparable with i-gel size 1 . Also, we found the feasibility of intubation with up to standard uncuffed $3.5 \mathrm{~mm}$ ETT through LMAclassic size 1.5 was statistically comparable with i-gel size 1.5. One of the greatest challenges encountered when intubating through these SADs occurs during the removal of the SAD. The main problem is the disappearance of the proximal end of the ETT due to similar lengths of the ETT and LMA. Cutting and shortening the LMA-classic, using a long guide wire or exchange catheter, using a laryngeal forceps to 
control the ETT and cutting the aperture bars of the LMA are among the major modifications published in the literature. $5,7,15,16$ We did not make any modifications in the SADs tested. Removal of the SAD was mostly not feasible, even following an easy intubation through LMA-proseal, LMA-classic and LMA-supreme. However, we did not encounter any problems during removal of the i-gel when intubation was possible.

During endotracheal intubation through an SAD, cuffed ETTs are favored by some physicians to avoid failure and exchange due to inappropriately fitted ETTs. Selecting a smallersized cuffed ETT could give the opportunity of easy insertion of an ETT and subsequent SAD removal with the ability to seal the trachea by inflating the cuff. ${ }^{6}$ However, SAD removal could be complicated due to insufficient space in the tiny lumen of the SAD to allow the passage of a pilot balloon. This condition is especially obvious for pediatric SADs up to size 2 and/or 2.5. ${ }^{6}$ Choosing SADs specifically designed as conduits with wider airway tubes and shorter lengths might be a solution to this problem. ${ }^{11}$ Correspondingly, we could not remove any of the tested SADs after intubation with cuffed ETTs, even with IDs of $2.5 \mathrm{~mm}$. Leaving the SAD in place during the whole operation or cutting the pilot balloon if appropriate could be alternative choices in such a situation.

Blind intubation through an SAD is not recommended due to the high incidence of laryngeal trauma, esophageal intubation and accidental dislodgement during SAD removal, especially in the pediatric population. ${ }^{3,17}$ Also, Jagannathan et al. $^{3}$ recorded $27 \%$ epiglottic downfolding, which obstructed the laryngeal view in their study conducted with air- $Q$ intubating laryngeal airway as a conduit for intubation in children. The fiberoptic grade of laryngeal view was negatively correlated with the weight of the children in that study. The literature suggests that epiglottic downfolding could be handled by articulating the FOB underneath the epiglottis, thus fiberoptic guidance should be strongly recommended during intubation through SADs in children. Although we tested the feasibility of intubation with standard ETTs with $2.5 \mathrm{~mm}$ ID through size 1 SADs in cases when intubation with
ETT with $3.0 \mathrm{~mm}$ ID was not possible, we do not recommend blind intubation through SADs in infants or newborns.

The study has some limitations. First of all, we only studied the devices on a standard intubation training manikin. The results may not directly apply to children with difficult airway. Secondly, none of the SADs tested in this study had a detachable proximal connector. This situation limited the passage of the pilot balloons of the ETTs. Selecting an SAD with a detachable proximal connector that is specially designed for tracheal intubation, such as air-Q could be a better choice for providing a functionally wider orifice for the passage of the pilot balloon. ${ }^{18}$ Lastly, we did not make any modifications in the SADs tested. The results might be different with preconcerted modifications of LMA-classic or LMA-proseal because collateral evidence supporting this theory could be found from several case reports in the literature. $5,7,15,16$

In conclusion; i-gel sizes 1 and 1.5 both performed better as conduits for fiberopticguided intubation compared with LMA-proseal, LMA-classic and LMA-supreme with most of the uncuffed ETTs investigated in our study. i-gel generally performed better than its own manufacturer recommendations as a conduit for intubation. However, the passage of cuffed ETTs was problematic with all types of studied SADs. Although the results of this manikin study suggest that the choice of i-gel as a conduit for intubation could be safer than LMA-classic, LMA-supreme and LMA-proseal; familiarity of use and the need for cuffed or uncuffed ETT may influence the choice of SAD.

\section{Ethics approval}

Ethical approval was not required for this manikin study.

\section{Acknowledgement}

We appreciate the work of Ergocen S, MSc for helping statistical analysis and Chapman D. for English editing.

\section{REFERENCES}

1. Sunder RA, Haile DT, Farrell PT, Sharma A. Pediatric airway management: current practices and future directions. Paediatr Anaesth 2012; 22: 1008-1015. 
2. Black AE, Flynn PE, Smith HL, Thomas ML, Wilkinson KA; Association of Pediatric Anaesthetists of Great Britain and Ireland. Development of a guideline for the management of the unanticipated difficult airway in pediatric practice. Paediatr Anaesth 2015; 25: 346-362.

3. Jagannathan N, Kozlowski RJ, Sohn LE, et al. A clinical evaluation of the intubating laryngeal airway as a conduit for tracheal intubation in children. Anesth Analg 2011; 112: 176-182.

4. Jagannathan N, Ramsey MA, White MC, Sohn L. An update on newer pediatric supraglottic airways with recommendations for clinical use. Paediatr Anaesth 2015; 25: 334-345.

5. Khan RM, Sharma PK, Kaul N. Laryngeal mask airwayaided fiberoptic tracheal intubation in infant--a modified technique. Paediatr Anaesth 2009; 19: 1131-1132.

6. Weiss M, Goldmann K. Caution when using cuffed tracheal tubes for fibreoptic intubation through paediatric-sized laryngeal mask airways. Acta Anaesthesiol Scand 2004; 48: 523

7. Xue FS, Luo MP, Liao X, Tang GZ. Measures to facilitate the classic laryngeal mask airway guided fiberoptic intubation in children with a difficult airway. Paediatr Anaesth 2008; 18: 1273-1275.

8. Kleine-Brueggeney M, Kotarlic M, Theiler L, Greif R. Limitations of pediatric supraglottic airway devices as conduits for intubation - an in vitro study. Can J Anaesth 2018; 65: 14-22.

9. Burjek NE, Nishisaki A, Fiadjoe JE, et al; PeDI Collaborative Investigators. Videolaryngoscopy versus fiber-optic intubation through a supraglottic airway in children with a difficult airway: an analysis from the multicenter pediatric difficult intubation registry. Anesthesiology 2017; 127: 432-440.

10. Sohn LE, Jagannathan N, Sequera-Ramos L, Sawardekar A, Schaldenbrand K, De Oliveira GS. A randomised comparison of free-handed vs air-QTM assisted fibreoptic-guided tracheal intubation in children. Anaesthesia 2014; 69: 723-728.
11. Jagannathan $\mathrm{N}$, Sohn L, Ramsey M, et al. A randomized comparison between the $\mathrm{i}-\mathrm{gel}^{\mathrm{TM}}$ and the air- $\mathrm{Q}^{\mathrm{TM}}$ supraglottic airways when used by anesthesiology trainees as conduits for tracheal intubation in children. Can J Anaesth 2015; 62: 587-594.

12. Lee JR, Kim MS, Kim JT, et al. A randomised trial comparing the $\mathrm{i}-\mathrm{gel}^{\mathrm{TM}}$ with the LMA Classic ${ }^{\mathrm{TM}}$ in children. Anaesthesia 2012; 67: 606-611.

13. Fukuhara A, Okutani R, Oda Y. A randomized comparison of the i-gel and the ProSeal laryngeal mask airway in pediatric patients: performance and fiberoptic findings. J Anesth 2013; 27: 1-6.

14. Swati, Panda NB, Rajeev S. Management of pilot balloon assembly malfunction in an armored endotracheal tube in a child. Paediatr Anaesth 2008; 18: 356-357.

15. Stricker PA, Fiadjoe JE, McCloskey JJ. Additional measures to facilitate fiberoptic guided tracheal intubation through the classic laryngeal mask airway in children. Paediatr Anaesth 2009; 19: 410-411.

16. Mauch J, Haas T, Weiss M. Distance from the laryngeal mask grip to endotracheal tube tip. A crucial point during fiberoptic intubation in children. Anaesthesist 2012; 61: 123-128.

17. Auden SM, Lerner GM. Blind intubation via the laryngeal mask: a word of caution. Paediatr Anaesth 2000; 10: 452 .

18. Jagannathan N, Sohn LE, Sawardekar A, et al. A randomized trial comparing the Ambu ${ }^{\circledR}$ Aura- ${ }^{\mathrm{TM}}$ with the air- $\mathrm{Q}^{\mathrm{TM}}$ intubating laryngeal airway as conduits for tracheal intubation in children. Paediatr Anaesth 2012; 22: 1197-1204 